(C) [2009] IEEE. Reprinted, with permission, from Basukala, Riyaj., Mohd Ramli Huda Adibah, and Sandrasegaran, Kumbesan 2009, 'Performance analysis of EXP/PF and M-LWDF in downlink 3GPP LTE system'2009 First Asian Himalayas International Conference on Internet. AH-ICl 2009, pp. 1-5.. This material is posted here with permission of the IEEE. Such permission of the IEEE does not in any way imply IEEE endorsement of any of the University of Technology, Sydney's products or services. Internal or personal use of this material is permitted. However, permission to reprint/republish this material for advertising or promotional purposes or for creating new collective works for resale or redistribution must be obtained from the IEEE by writing to pubs-permissions@ieee.org. By choosing to view this document, you agree to all provisions of the copyright laws protecting it. 


\title{
Performance Analysis of EXP/PF and M-LWDF in Downlink 3GPP LTE System
}

\author{
R. Basukala ${ }^{\# 1}$, H.A. Mohd Ramli ${ }^{\# 2}$, and K. Sandrasegaran ${ }^{\# 3}$, Member, IEEE \\ Faculty of Engineering and Information Technology, \\ University of Technology Sydney, \\ 15 Broadway, Ultimo, \\ NSW 2007, Australia \\ ${ }^{1}$ Riyaj.Basukala@uts.edu.au \\ $\left[{ }^{2}\right.$ HudaAdibah.MohdRamli, ${ }^{3}$ kumbes] @eng.uts.edu.au \\ Telephone: +61295142428 \\ Fax: +61 295142435
}

\begin{abstract}
This paper investigates the performance of exponential/proportional fair (EXP/PF) and maximum-largest weighted delay first (M-LWDF) scheduling algorithms in the third generation partnership project long term evolution (3GPP LTE) providing packet-switched multimedia services. It, then, identifies a suitable packet scheduling algorithm on a basis of their performance evaluation. The performance evaluation is conducted in terms of system throughput, average real time (RT) and non-real time (NRT) throughput, packet loss for RT service and fairness for NRT service. The video streaming traffic is used to model the RT service. While, the web-browsing traffic is modelled for NRT service. Simulation results show that at lower load MLWDF algorithm provides better performance than EXP/PF while as the load increases the EXP/PF gives better performance.
\end{abstract}

Keywords - Packet scheduling, 3GPP LTE, QoS, multimedia services, EXP/PF, M-LWDF.

\section{INTRODUCTION}

LTE is a new radio access technology that uses orthogonal frequency division multiple access (OFDMA) in the downlink. OFDMA is an access technology that divides the available bandwidth into multiple narrow-band subcarriers. A portion of the available sub-carriers will be allocated to a user based on the user's requirements, current load and system configuration. OFDMA promises improved system capabilities by providing higher data rates, supporting multi-user diversity and being resistant to frequency selective fading of the radio channels by using different sub-carriers according to the channel conditions. The LTE Radio Network Architecture consists of only eNodeBs that perform all radio resource management (RRM) functions.

Packet-switched multimedia applications will become a norm in future wireless communications and their quality of service (QoS) requirements need to be guaranteed. These applications can be divided into RT and NRT services. The RT services may either be delay sensitive (e.g. voice-overIP), loss sensitive (e.g. video streaming) or both (e.g. video conferencing). The NRT services do not have strict requirements and are best-effort. They are served when there are spare resources available. In this paper, to represent multimedia traffic scenario, a video streaming service and web browsing service are considered. To ensure that the QoS requirements of RT video streaming users are satisfied, the packet loss has to be minimized and a minimum throughput must be maintained. RT packet loss is a performance metric that is related to the packet delay.

Packet scheduling is one of the RRM mechanisms and it is responsible for intelligent selection of a user's packets to use the available system resources such that specified performance metrics are satisfied. The need to support diverse QoS requirements of different applications whilst maximizing system throughput is one of the major challenges in the design of packet scheduling algorithms in the downlink 3GPP LTE system

The aim of this paper is to investigate the performance of two of the well known packet scheduling algorithms, EXP/PF and M-LWDF, developed for single carrier wireless systems in provisioning of multimedia services for downlink LTE system. This paper contributes to the identification of a suitable packet scheduling algorithm for use in the downlink 3GPP LTE system for supporting multimedia services with video streaming and web browsing.

The remainder of this paper is organized as follows. Section II describes system model in the downlink 3GPP LTE system followed by discussions on packet scheduling algorithms in Section III. The simulation environment is discussed in Section IV while Section V discusses the traffic models used. Section VI contains the results of the simulation and Section VII concludes the paper.

\section{DOWNLINK 3 GPP LTE SYSTEM MODEL}

The resource that is allocated to a user in the downlink 3GPP LTE system contains both frequency and time domains and is called a resource block (RB). In the frequency domain, the RB consists of 12 sub-carriers (total bandwidth of $180 \mathrm{kHz}$ ) and in the time domain it is made up 
of one time slot of $0.5 \mathrm{~ms}$ duration. A time slot consists of 7 OFDM symbols [1].

The architecture of 3GPP LTE system consists of e-Node Bs where packet scheduling is performed along with other RRM mechanisms. In this paper, a cell of $5 \mathrm{MHz}$ bandwidth with $25 \mathrm{RBs}$ and $2 \mathrm{GHz}$ carrier frequency is modeled. The e-Node $\mathrm{B}$ is fixed at the centre of the cell and it controls all the available RBs. These RBs are to be shared by all users within the cell. Scheduling in this system is performed at 1 ms interval (transmit time interval, TTI) which consists of 2 time slots. Two consecutive RBs (in time domain) can be assigned to a user for each TTI. The system parameters of the downlink 3GPP LTE system are given in Table I.

Users report their instantaneous downlink channel conditions (e.g. signal-to-noise-ratio, SNR) to serving eNode B at each TTI. The received instantaneous downlink SNR values of each user vary on each sub-carrier and at each TTI due to the frequency-selective fading nature of multi-path propagation and time-selective fading nature due to user movement respectively [2, 3]. The reported instantaneous downlink SNR value is used to determine the downlink data rate, which gives the number of bits in two consecutive RBs that a user can support at each TTI.

The approach proposed in [4] is used to compute the number of bits per symbol of user $i$ at time $t$ on a sub-carrier

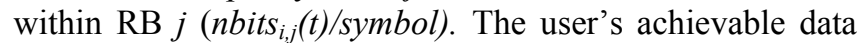
rate $\left(d r_{i}(t)\right)$ at time $t$ on two consecutive RBs are determined using:

$d r_{i}(t)=\frac{\text { nbits }_{i, j}(t)}{\text { symbol }} * \frac{\text { nsymbols }}{\text { slot }} * \frac{\text { nslots }}{T T I} * \frac{n s c}{R B}$

where nsymbols/slot is the number of symbols per slot, $n s l o t / T T I$ is the number of slots per TTI and $n s c / R B$ is the number of sub-carriers per RB. The minimum instantaneous downlink SNR value and the associated achievable data rate used are given in Table II.

Each user is assigned a buffer at e-Node B. Packets arriving into the buffer are time stamped and queued for transmission based on a first-in-first-out (FIFO) basis. For each packet in the queue at the e-Node buffer, the head of line (HOL) packet delay (time difference between the current time and the arrival time of a packet) is computed. If the HOL packet delay exceeds a specified threshold, then packets are discarded.

TABLE I

3GPp Lte Downlink System Parameters

\begin{tabular}{|cc|}
\hline Parameters & Values \\
\hline Carrier Frequency & $2 \mathrm{GHz}$ \\
\hline Bandwidth & $5 \mathrm{MHz}$ \\
\hline Number of Sub-carriers & 300 \\
\hline Number of RBs & 25 \\
\hline Number of Sub-carriers per RB & 12 \\
\hline Sub-Carrier Spacing & $15 \mathrm{kHz}$ \\
\hline Slot Duration & $0.5 \mathrm{~ms}$ \\
\hline Scheduling Time (TTI) & $1 \mathrm{~ms}$ \\
\hline Number of OFDM Symbols per Slot & 7 \\
\hline
\end{tabular}

TABLE II

Instantaneous Downlink SnR To Data Rate Mapping Table

\begin{tabular}{|ccc|}
\hline $\begin{array}{c}\text { Minimum } \\
\text { Instantaneous } \\
\text { Downlink SNR } \\
\text { Value (dB) }\end{array}$ & $\begin{array}{c}\text { Modulation and } \\
\text { Coding }\end{array}$ & $\begin{array}{c}\text { Data Rate } \\
\text { (kbps) }\end{array}$ \\
\hline 1.7 & QPSK (1/2) & 168 \\
\hline 3.7 & QPSK (2/3) & 224 \\
\hline 4.5 & QPSK (3/4) & 252 \\
\hline 7.2 & 16 QAM (1/2) & 336 \\
\hline 9.5 & 16 QAM (2/3) & 448 \\
\hline 10.7 & 16 QAM (3/4) & 504 \\
\hline 14.8 & 64 QAM (2/3) & 672 \\
\hline 16.1 & 64 QAM (3/4) & 756 \\
\hline
\end{tabular}

The packet scheduler determines a user's priority based on a scheduling algorithm. These algorithms use certain scheduling criteria (e.g. channel conditions, HOL packet delays, buffer status, service types, etc.) when making scheduling decisions. Once a user has been selected for transmission, the number of bits to be transmitted is based on the user's reported instantaneous downlink SNR value. At each TTI, a user may be allocated one or more RBs based on packet scheduling algorithm being used (as shown in Fig. 1).

\section{PACKET SHEDULING ALGORITHMS}

The packet scheduling algorithms to be used in 3GPP LTE should aim to maximize throughput, satisfy users' QoS and provide good fairness to NRT users. The algorithms that are considered in this paper are the packet scheduling algorithms that were developed for single carrier wireless systems and good candidates for use in 3GPP LTE.

According to [5-7], system throughput can be maximized if the packet scheduler utilizes the reported instantaneous downlink channel conditions from the users when making scheduling decisions. However, scheduling decisions that rely on channel conditions only are insufficient to support multimedia applications due to their strict delay requirements. Hence, several packet scheduling algorithms that satisfy this new requirement have been developed.

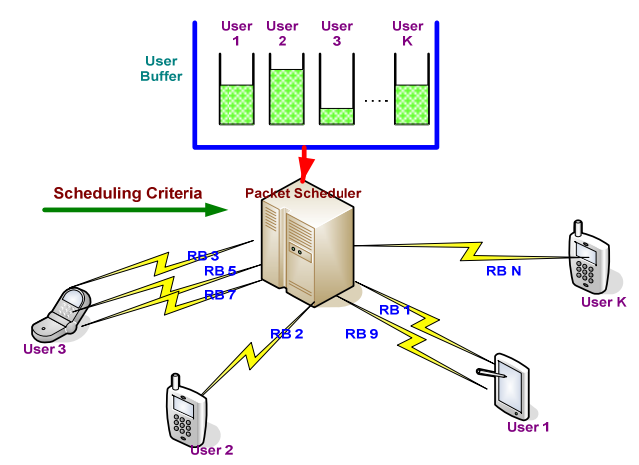

Fig. 1. General Packet Scheduling Model in the Downlink 3GPP LTE System 
The maximum-largest weighted delay first (M-LWDF) [8] is an algorithm designed to support multiple data users with varying QoS requirements within CDMA-HDR system. A user is selected based on the following equations.

$k=\arg \max a_{i} W_{i}(t) \frac{r_{i}(t)}{R_{i}(t)}$

$a_{i}=-\frac{\left(\log \delta_{i}\right)}{\tau_{i}}$

and:

$$
R_{i}(t)=\left(1-\frac{1}{t_{c}}\right) * R_{i}(t-1)+\frac{1}{t_{c}} * r_{i}(t-1)
$$

where $W_{i}(t)$ is the HOL packet delay of user $i$ at time $t, \tau_{i}$ is the delay threshold of user $i$ 's packets (different for RT and NRT packets) and $\delta_{i}$ is the maximum probability for HOL packet delay of user $i$ to exceed the delay threshold of user $i$. $r_{i}(t) \& R_{i}(t)$ are terms derived from proportional fair (PF) scheduling algorithm [9]. $r_{i}(t)$ is the achievable data rate of user $i, R_{i}(t)$ is the average data rate of user $i$ over a time window $\left(t_{c}\right)$ of an appropriate size. This update window size determines between maximizing throughput and satisfying fairness of each user in PF algorithm. M-LWDF algorithm thus incorporates HOL packet delay together with PF properties (e.g. the ratio of achievable data rate to the average data rate) when determining users' priority. MLWDF prioritizes the user with higher HOL packet delay and better channel conditions relative to its average levels.

The exponential/proportional fair (EXP/PF) $[10,11]$ algorithm was developed to support multimedia applications in an adaptive modulation and coding and time division multiplexing (AMC/TDM) system. A user in AMC/TDM system can either belong to a RT service or a NRT service. Therefore, the EXP/PF equation to be used for a user depends on the user's service type. The metric $k$ is computed for each user for RT and NRT services using the following equation:

$$
k=\arg \max \left\{\begin{array}{l}
\exp \left(\frac{a_{i} W_{i}(t)-\overline{a W(t)}}{1+\sqrt{a W(t)}}\right) \frac{r_{i}(t)}{R_{i}(t)} \ldots . i \in R T \\
\frac{w(t)}{M(t)} \frac{r_{i}(t)}{R_{i}(t)} \ldots \ldots \ldots \ldots \ldots \ldots \ldots \ldots . . . i \in N R T
\end{array}\right.
$$

and:

$$
\begin{aligned}
& \overline{a W(t)}=\frac{1}{N_{R T}} \sum_{i \in R T} a_{i} W_{i}(t) \\
& w(t)=\left\{\begin{array}{l}
w(t-1)-\varepsilon \ldots \ldots . W_{\max }>\tau_{\max } \\
w(t-1)+\frac{\varepsilon}{k} \ldots . . W_{\max }<\tau_{\max }
\end{array}\right.
\end{aligned}
$$

where $M(t)$ is the average number of RT packets waiting at e-Node B buffer at time $t, \varepsilon$ and $k$ are constants, $W_{\max }$ is the maximum HOL packet delay of all RT service users and $\tau_{\max }$ (same as RT $\tau_{i}$ in (3)) is the maximum delay constraint of RT service users. In the EXP/PF algorithm, RT users receive increased priority than NRT users when their HOL packet delays are approaching the delay deadline.

\section{SIMULATION ENVIRONMENT}

In this paper a single hexagonal cell of radius $150 \mathrm{~m}$ with the downlink LTE parameters as discussed in Section II is modeled. There are $50 \%$ video streaming and $50 \%$ web browsing users within the cell and they are uniformly distributed within the serving eNodeB. Users are constantly moving at the speeds between $1-100 \mathrm{~km} / \mathrm{h}$ in random directions. A wrap-around method [12] is employed at the cell boundary to ensure users always remain within the simulated area. It is worthwhile to mention that several assumptions are made in this paper due to time limitations as well as in order to reduce complexity of the system simulation. These assumptions are consistent with other works [13-14].

It is assumed that users report their instantaneous downlink SNR values on each RB to the serving eNodeB and this reporting is assumed to be error-free and delay-free. The reported instantaneous downlink SNR values are determined based on sub-carrier located at the centre frequency of each RB. It is also assumed that all subcarriers are used for data transmission and equal downlink transmit power is allocated on each sub-carrier.

Pathloss in urban environment [15], shadow fading [16] and multi-path fading [17] are used to determine the channel gain and hence the instantaneous downlink SNR value of each user on each RB. It is assumed in this paper that at any time instant, multi-path values vary on each $\mathrm{RB}$ whereas pathloss and shadow fading are fixed on each RB. The channel gain $\left(\operatorname{Gain}_{i, j}(t)\right)$ of user $i$ on RB $j$ at time $t$ is computed using:

$\left.\operatorname{Gain}_{i, j}(t)=10^{\left(\frac{\text { pl }_{i}(t)}{10}\right)} * 10^{\left(\frac{\xi_{i}(t)}{10}\right)} * 10^{\left(\frac{\text { mpath }}{10}(t)\right.}\right)$

where, $p l_{i}(t)$ and $\xi_{\mathrm{i}}(t)$ are the pathloss and shadow fading gain of user $i$ at time $t$, respectively and mpath $_{i, j}(t)$ is the multi-path fading gain of user $i$ on RB $j$ at time $t$. From the computed channel gain, the instantaneous downlink SNR value of user $i$ on RB $j$ at time $t\left(\gamma_{i, j}(t)\right)$ is computed using the approach proposed in [18] as given below:

$\gamma_{i, j}(t)=\frac{P_{\text {total }} * \operatorname{Gain}_{i, j}(t)}{N\left(I+N_{o}\right)}$

where, $P_{\text {total }}$ is the total eNodeB downlink transmit power, $N$ is the number of available RBs, $N_{o}$ is the thermal noise and $I$ is the inter-cell interference. The inter-cell interference is dependent on user's location and since the simulation time is very small, the user's location does not vary much and so the inter-cell interference is assumed constant.

The performance of each algorithm is evaluated based on system throughput, average throughput, PLR (for RT traffic) and fairness (for NRT traffic) as defined below: 
systemthroughput $=\frac{1}{T} \sum_{i=1}^{K} \sum_{t=1}^{T}$ ptransmit $_{i}(t)$

PLR $=\frac{\sum_{i=1}^{K} \sum_{t=1}^{T} \text { pdiscard }_{i}(t)}{\sum_{i=1}^{K} \sum_{t=1}^{T} \text { psize }_{i}(t)}$

fairness $=1-\frac{\text { ptotaltransmit }_{\max }-\text { ptotaltransmit }_{\min }}{S^{K}}$

$$
\sum_{i=1}^{K} \sum_{t=1}^{T} \operatorname{psize}_{i}(t)
$$

averagethroughput $_{i}=\frac{1}{T} \sum_{t=1}^{T}$ ptransmit $_{i}(t)$

where $\operatorname{ptransmit}_{i}(t)$, $\operatorname{pdiscard}_{i}(t)$ and $\operatorname{psize}_{i}(t)$ are the size of transmitted packets, the size of discarded packets and the size of all packets that have arrived into eNodeB buffer of user $i$ at time $t$, respectively, ptotaltransmit $t_{\max }$ and ptotaltransmit $_{\min }$ are the total size of the transmitted packets of the most and the least served users, respectively, $K$ is the total number of users and $T$ is the total simulation time.

\section{TRAFFIC MODELS}

A video streaming service with $128 \mathrm{kbps}$ source video data rate is used in the simulation and its parameters are shown in Table III. The threshold for HOL packet delay for this service is set to $20 \mathrm{~ms}$ which is the maximum waiting time of the packet at the serving eNodeB buffer [19]. The eNodeB buffer of each user is assumed to be infinite and packet is considered lost when it is discarded, not due to buffer overflow. For simulation purposes, it is assumed that the de-jitter buffer, with window size for video streaming service of 5 seconds, at the user end is initially full [20]. Assuming that the mean run time of a video streaming is about 23 seconds, it can be calculated that to run a video streaming for that duration without the buffer running dry, a minimum throughput of $100 \mathrm{kbps}$ has to be maintained for the user across the air interface. Table IV shows the parameters used to generate NRT service of web browsing. There is no minimum throughput requirement for this traffic as it is served as best effort traffic.

TABLE III

PARAmeters of Video Streaming ApPlication [20]

\begin{tabular}{|ccc|}
\hline $\begin{array}{c}\text { Information } \\
\text { types }\end{array}$ & Distribution & $\begin{array}{c}\text { Distribution } \\
\text { Parameters }\end{array}$ \\
\hline $\begin{array}{c}\text { Inter-arrival time } \\
\text { between the } \\
\text { beginning of } \\
\text { successive frames }\end{array}$ & $\begin{array}{c}\text { Deterministic (Based } \\
\text { on 20fps) }\end{array}$ & $50 \mathrm{~ms}$ \\
\hline $\begin{array}{c}\text { Number of } \\
\text { packets (slices) in a } \\
\text { frame }\end{array}$ & Deterministic & 8 \\
\hline $\begin{array}{c}\text { Packet (slice) size } \\
\text { Truncated Pareto } \\
\text { (Mean=50bytes, } \\
\text { max=125bytes) }\end{array}$ & $\begin{array}{c}\mathrm{K}=40 \mathrm{bytes}, \\
\alpha=1.2\end{array}$ \\
\hline $\begin{array}{c}\text { Inter-arrival time } \\
\text { between packets } \\
\text { (slices) in a frame }\end{array}$ & $\begin{array}{c}\text { Truncated Pareto } \\
(\text { Mean=6ms, } \\
\text { Max=12.5ms) }\end{array}$ & $\begin{array}{c}\mathrm{K}=2.5 \mathrm{~ms}, \\
\alpha=1.2\end{array}$ \\
\hline
\end{tabular}

TABLE IV

Parameters of Web Browsing ApPlication [20]

\begin{tabular}{|c|c|c|}
\hline Component & Distribution & $\begin{array}{l}\text { Distribution } \\
\text { Parameters }\end{array}$ \\
\hline $\begin{array}{l}\text { Main object size } \\
\qquad\left(\mathrm{S}_{\mathrm{M}}\right)\end{array}$ & $\begin{array}{l}\text { Truncated Lognormal } \\
\text { (Min=100 bytes, } \\
\text { Max }=20 \text { Kbytes) }\end{array}$ & $\begin{array}{l}\sigma=25032 \\
\text { bytes, } \\
\mu=10710 \\
\text { bytes }\end{array}$ \\
\hline $\begin{array}{l}\text { Embedded object } \\
\quad \text { size }\left(\mathrm{S}_{\mathrm{E}}\right)\end{array}$ & $\begin{array}{c}\text { Truncated Lognormal } \\
(\text { Min }=50 \text { bytes, Max }=20 \\
\text { Kbytes })\end{array}$ & $\begin{array}{l}\sigma=126168 \\
\text { bytes, } \\
\mu=7758 \\
\text { bytes }\end{array}$ \\
\hline $\begin{array}{c}\text { Number of } \\
\text { embedded objects per } \\
\text { page }\left(\mathrm{N}_{\mathrm{d}}\right)\end{array}$ & $\begin{array}{l}\text { Truncated Pareto } \\
(\text { Mean=5.64, } \\
\text { Max=53) }\end{array}$ & $\begin{array}{c}\mathrm{K}=2, \mathrm{~m}=55, \\
\alpha=1.1\end{array}$ \\
\hline Parsing time $\left(\mathrm{T}_{\mathrm{p}}\right)$ & $\begin{array}{c}\text { Exponential } \\
(\mathrm{Mean}=0.13 \mathrm{sec})\end{array}$ & $\chi=7.69$ \\
\hline Reading time $\left(\mathrm{D}_{\mathrm{pc}}\right)$ & $\begin{array}{c}\text { Exponential } \\
(\text { Mean=30 sec) }\end{array}$ & $\chi=0.33$ \\
\hline
\end{tabular}

\section{SiMULATION RESULTS}

Fig.2 shows overall system throughput while Fig. 3 displays average throughputs for RT and NRT service users for both algorithms considered. System throughput graph shown in Fig. 2 demonstrates that for lower number of users both EXP/PF and M-LWDF show similar throughput performances but as the users increase M-LWDF provides higher system throughput. This can be explained using Fig. 3 , which shows that M-LWDF provides higher average throughput for NRT users as compared to EXP/PF, thus lowering the system throughput for the latter algorithm. As EXP/PF dynamically adjusts NRT priority $(5,7)$, with increase in number of users, it maintains the required RT throughput (100 kbps) for up to 260 users by sacrificing the resources for NRT users. While from Fig. 3, M-LWDF can only provide throughput guarantee to RT service for up to 160 users.

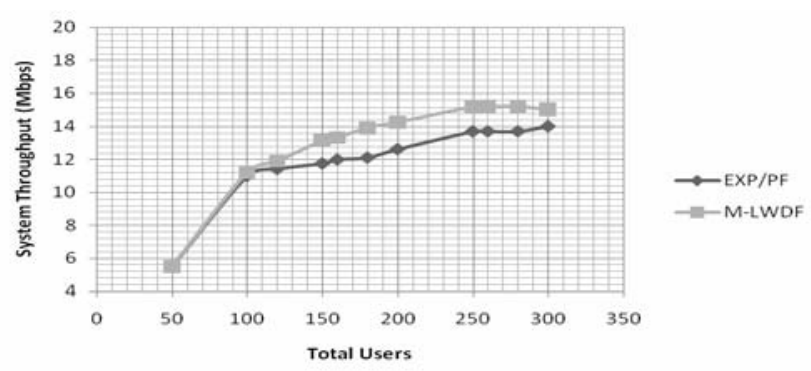

Fig. 2. System Throughput vs. Number of User

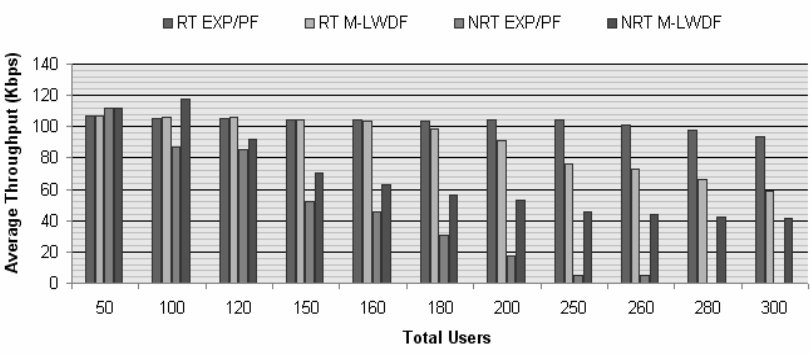

Fig. 3. Average Throughput vs. Number of Users 


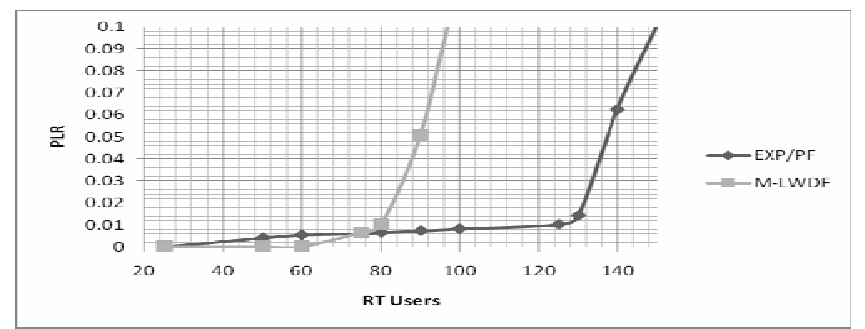

Fig. 4. Packet Loss Ratio for RT users vs. Number of RT Users

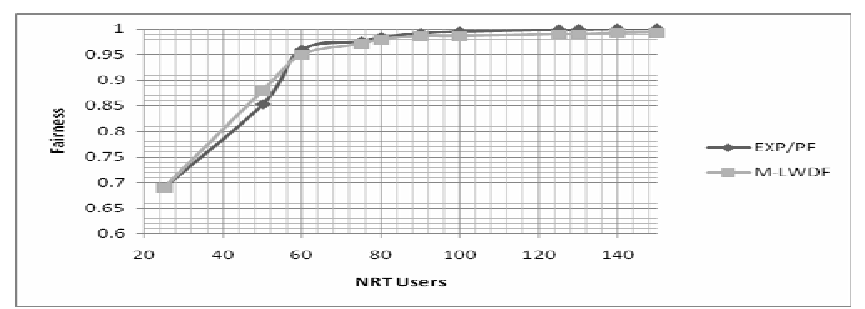

Fig. 5. Fairness for NRT Users vs. Number of NRT Users

RT users' average throughput in M-LWDF degrades faster as it only considers HOL delay and relative channel conditions and fails to increase priority for RT users when their packets' delay deadline is approaching. The PLR requirement for video streaming service of $1 \%$ [21] is maintained by EXP/PF algorithm for about $125 \mathrm{RT}$ users while M-LWDF can only meet the requirement if there are 80 or less RT users in the system (Fig. 4). More packet loss for real time users in M-LWDF is explained by its decreasing average RT throughput and higher NRT throughput as compared to EXP/PF algorithm in Fig. 3. This is mainly due to the failure of M-LWDF algorithm to dynamically adjust the priorities for RT and NRT traffic according to the strict delay requirements of RT traffic. Lastly, Fig. 5 shows that the fairness performance of both algorithms increases with increase in users and they provide similar fairness performance for NRT service.

\section{CONCLUSION}

This paper mainly focused on the performance of EXP/PF and M-LWDF packet scheduling algorithms in downlink LTE system for providing basic multimedia services consisting of RT video streaming service and NRT web browsing service. The simulation results showed that in the downlink 3GPP LTE system supporting those services (in $50 \% \mathrm{RT}$ and $50 \%$ NRT scenario) in a $150 \mathrm{~m}$ radius cell, MLWDF algorithm gives better performance as compared to $\mathrm{EXP} / \mathrm{PF}$ for lower loads with users' number ranging from 50 to 160 . Required PLR of $1 \%$ and average RT throughput of $100 \mathrm{kbps}$ are maintained by M-LWDF in that range providing better system throughput. However, as the load increases, EXP/PF showed the sustained performance in terms of average RT throughput and PLR requirements for RT service loads of up to 250 users. However, some system throughput is sacrificed in this particular scenario when $\mathrm{EXP} / \mathrm{PF}$ is used, as it prioritizes $\mathrm{RT}$ video streaming whose throughput is capped by video source encoder data rate. On the whole, it is recommended to employ simpler M-LWDF algorithm for lower loads and use computationally complex $\mathrm{EXP} / \mathrm{PF}$ algorithm for higher loads in downlink LTE system supporting multimedia services.

\section{REFERENCES}

[1] J. Zyren, "Overview of 3GPP Long Term Evolution Physical Layer," freescale semiconductor, 2007.

[2] J. Gross, H. Karl, F. Fitzek, and A. Wolisz, "Comparison of Heuristic and Optimal Subcarrier Assignment Algorithms," in Proceedings of International Conference on Wireless Networks, 2003.

[3] A. K. F. Khattab and K. M. F. Elsayed, "Opportunistic Scheduling of Delay Sensitive Traffic in OFDMA-based Wireless Networks," in International Symposium on a World of Wireless, Mobile and Multimedia Networks, 2006, pp. 10 pp.-288.

[4] X. Qiu and K. Chawla, "On the Performance of Adaptive Modulation in Cellular Systems," in IEEE Transactions on Communications. vol. 47, 1999, pp. 884-895.

[5] A. Gyasi-Agyei and S.-L. Kim, "Comparison of Opportunistic Scheduling Policies in Time-Slotted AMC Wireless Networks," in 1st International Symposium on Wireless Pervasive Computing, 2006.

[6] L. Yan and Y. Guangxin, "Channel-Adapted and Buffer-Aware Packet Scheduling in LTE Wireless Communication System," in 4th International Conference on Wireless Communications, Networking and Mobile Computing, 2008, pp. 1-4.

[7] Y. J. Zhang and S. C. Liew, "Link-Adaptive Largest-WeightedThroughput Packet Scheduling for Real-Time Traffics in Wireless OFDM Networks," in IEEE GLOBECOM, 2005.

[8] M. Andrews, K. Kumaran, K. Ramanan, A. Stolyar, P. Whiting, and R. Vijayakumar, "Providing Quality of Service over a Shared Wireless Link," IEEE Communications Magazine, vol. 39, pp. 150154, Feb. 2001

[9] A. Jalali, R. Padovani, and R. Pankaj, "Data Throughput of CDMAHDR a High Efficiency-High Data Rate Personal Communication Wireless System," in IEEE 51st Vehicular Technology Conference Proceedings, Tokyo, 2000, pp. 1854-1858.

[10] J.-H. Rhee, J. M. Holtzman, and D. K. Kim, "Scheduling of Real/Non-real Time Services: Adaptive EXP/PF Algorithm," in The 57th IEEE Semiannual Vehicular Technology Conference. vol. 1, 2003, pp. 462-466.

[11] J.-H. Rhee, J. M. Holtzman, and D. K. Kim, "Performance Analysis of the Adaptive EXP/PF Channel Scheduler in an AMC/TDM System," IEEE Communications Letters, vol. 8, pp. 4978-4980, Aug. 2004.

[12] A. G. Orozco Lugo, F. A. Cruz Prez, and G. Hernandez Valdez, "Investigating the Boundary Effect of a Multimedia TDMA Personal Mobile Communication Network Simulation," in IEEE VTS 54th Vehicular Technology Conference, 2001, pp. 2740-2744 vol.4.

[13] L. Xiantao, L. Guangyi, W. Ying, and Z. Ping, "Downlink Packet Scheduling for Real-Time Traffic in Multi-User OFDMA System," in IEEE 64th Vehicular Technology Conference, 2006, pp. 1 - 5

[14] N. Xu, T. Zou, Y. Wang, and P. Zhang, "A MC-GMR Scheduler for Shared Data Channel in 3GPP LTE System," in IEEE 64th Vehicular Technology Conference, 2006, pp. 1-5.

[15] T. S. Rappaport, Wireless Communications: Principles and Practice, 3rd ed., Prentice Hall PTR, 2003, pp. 153-154.

[16] M. Gudmundson, "Correlation Model for Shadow Fading in Mobile Radio Systems," in Electronics Letters. vol. 27, 1991, pp. 2145-2146.

[17] C. Komninakis, "A Fast and Accurate Rayleigh Fading Simulator," in IEEE Globecom San Francisco, CA, 2003.

[18] K. Kim, G.-M. Yeo, B.-H. Ryu, and K. Chang, "Interference Analysis and Subchannel Allocation Schemes in Tri-Sectored OFDMA Systems," in IEEE 66th Vehicular Technology Conference, pp. 1857$1861,2007$.

[19] L. Senthikumar and V. Sankaranayanan, "QoS Provisioning Through a Delay Based Endpoint Admission Control for Diffserv Network," Information Technology Journal, vol. 3, pp. 448-453, 2006.

[20] 3GPP2, "CDMA-2000 Evaluation Methodology," December 10, 2004.

[21] T. Janevski, Traffic Analysis and Design of Wireless IP Networks. Norwood, MA: Artech House, 2003. 